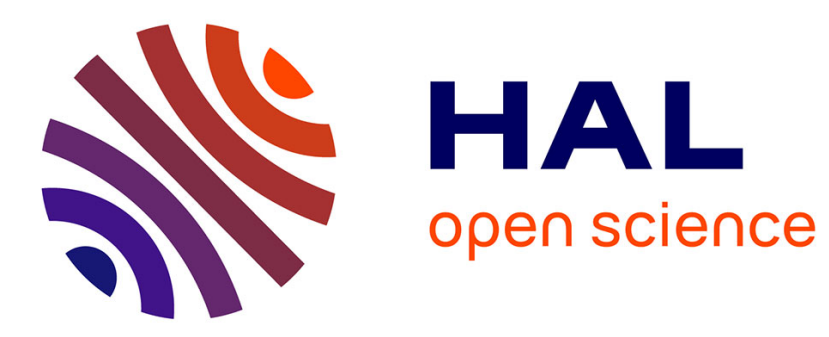

\title{
Marchands florentins et trafics caravaniers : une connexion à travers les négociants juifs dans la Méditerranée du XVe siècle
}

\author{
Ingrid Houssaye Michienzi
}

\section{- To cite this version:}

Ingrid Houssaye Michienzi. Marchands florentins et trafics caravaniers: une connexion à travers les négociants juifs dans la Méditerranée du XVe siècle. Histoire monde, jeux d'échelles et espaces connectés, Publications de la Sorbonne, pp.147-160, 2017, Actes du 47e congrès de la SHMESP. hal02386307

\section{HAL Id: hal-02386307 \\ https://hal.science/hal-02386307}

Submitted on 20 Sep 2021

HAL is a multi-disciplinary open access archive for the deposit and dissemination of scientific research documents, whether they are published or not. The documents may come from teaching and research institutions in France or abroad, or from public or private research centers.
L'archive ouverte pluridisciplinaire HAL, est destinée au dépôt et à la diffusion de documents scientifiques de niveau recherche, publiés ou non, émanant des établissements d'enseignement et de recherche français ou étrangers, des laboratoires publics ou privés. 


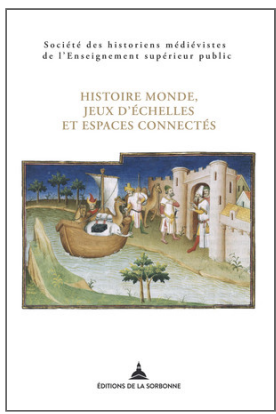

Société des historiens médiévistes de l'Enseignement supérieur public (dir.)

Histoire monde, jeux d'échelles et espaces connectés XLVII Congrès de la SHMESP (Arras, 26-29 mai 2016)

Éditions de la Sorbonne

\section{Marchands florentins et trafics caravaniers : une connexion à travers les négociants juifs dans la Méditerranée du XV ${ }^{\mathrm{e}}$ siècle}

Florentine Merchants and Caravan Trade: A Connection through the Jewish Merchants in the Mediterranean in the 15th Century

Ingrid Houssaye Michienzi

DOI : 10.4000/books.psorbonne.24819

Éditeur : Éditions de la Sorbonne

Lieu d'édition : Éditions de la Sorbonne

Année d'édition : 2017

Date de mise en ligne : 18 juillet 2019

Collection : Histoire ancienne et médiévale

ISBN électronique : 9791035101312

\section{Q books}

http://books.openedition.org

Référence électronique

HOUSSAYE MICHIENZI, Ingrid. Marchands florentins et trafics caravaniers : une connexion à travers les négociants juifs dans la Méditerranée du xve siècle In : Histoire monde, jeux d'échelles et espaces connectés : XLVII Congrès de la SHMESP (Arras, 26-29 mai 2016) [en ligne]. Paris : Éditions de la Sorbonne, 2017 (généré le 27 mars 2020). Disponible sur Internet : <http://books.openedition.org/ psorbonne/24819>. ISBN : 9791035101312 . DOI : https://doi.org/10.4000/books.psorbonne.24819. 


\title{
Marchands florentins et trafics caravaniers : une connexion à travers les négociants juifs dans la Méditerranée $\mathrm{du} \mathrm{XV}^{\mathrm{e}}$ siècle
}

\author{
Ingrid Houssaye Michienzi
}

L'objectif du présent article est d'explorer comment les marchands florentins purent avoir accès aux trafics caravaniers transsahariens et du Moyen-Orient grâce à des relations d'affaires tissées notamment avec des négociants et artisans juifs et juifs convertis. Les documents utilisés sont issus directement de la pratique des affaires et sont composés à la fois de lettres marchandes et de registres de comptes dans lesquels les modus operandi apparaissent très clairement, consentant la mise en lumière des dynamiques d'entreprises et des stratégies des acteurs. Ils lèvent également le voile sur un monde de marchands et de drapiers, des personnes décisives dans l'échange mais peu documentées par ailleurs. L'étude se focalise ainsi sur ces intermédiaires qui avaient une fonction spécifique dans la transaction marchande et qui constituaient un rouage majeur dans le fonctionnement de l'économie internationale ${ }^{1}$.

\footnotetext{
1. Nous renvoyons à diverses études récentes qui se sont penchées sur la notion d'" intermédiaires ", dans un contexte méditerranéen, ou dans le cadre de l'histoire globale à l'époque médiévale et moderne. Voir notamment S.D. Aslanian, From the Indian Ocean to the Mediterranean. The Global Trade Networks of Armenian Merchants from New Julfa, Berkeley, 2011; Renaissance GoBetweens: Cultural Exchange in Early Modern Europe, éd. A. Höfele, W. von Koppenfels, Berlin, 2005; Atlantic Diasporas. Jews, Conversos, and Crypto-Jews in the Age of Mercantilism, 1500-1800, éd. R. L. Kagan, P. D. Morgan, Baltimore, 2009; Double Agents: Cultural and Political Brokerage in Early Modern Europe, éd. M. Keblusex, B. Noldus, Leyde, 2011; L. Nussdorfer, Brokers of Public Trust: Notaries in Early Modern Rome, Baltimore, 2009; K. L. Reyerson, The Art of the Deal: Intermediaries of Trade in Medieval Montpellier, Leyde, 2002; N. Rothмan, Brokering Empire: Trans-Imperial Subjects between Venice and Istanbul, Ithaca, 2011; S. Schaffer, The Brokered World. Go-Betweens and Global Intelligence, 1770-1820, éd. L. Roberts, K. RaJ, J. Delbourgo, Sagamore Beach (MA), 2009; S. Subrahmanyam, Three Ways to Be Alien. Travails and Encounters in the Early Modern World, Waltham, 2011; F. Trivellato, The Familiarity of Strangers. The Sephardic Diaspora, Livorno, and Cross-Cultural Trade in the Early Modern Period, New Haven/Londres, 2009, trad. G. Calafat, Corail contre diamants. Réseaux marchands, diaspora sépharade et commerce
} 
Ces deux cas d'étude se situent aux deux extrémités du $\mathrm{Xv}^{\mathrm{e}}$ siècle, une période durant laquelle l'État florentin devint un acteur d'envergure en Méditerranée. En 1421, Florence acquit une façade maritime désirée depuis fort longtemps, et commença à mettre sur pied une organisation de voyages de galées similaire à celle des mude vénitiennes ${ }^{2}$. Le développement d'une flotte et de convois maritimes desservant la Méditerranée et l'océan Atlantique, du Levant jusqu'en Flandre, permit aux négociants florentins de ne plus dépendre de l'affrètement de navires d'autres puissances maritimes. De même, les ambassades, notamment à Tunis, à Alexandrie et à Constantinople, suivies de la promulgation de traités de paix et de commerce, rendirent inutile la nécessité de se cacher derrière le pavillon d'autres nations pour commercer ${ }^{3}$. Ce fut ainsi la fin d'une longue période durant laquelle, dans le domaine des transports maritimes et de la diplomatie, les marchands florentins durent exclusivement s'appuyer sur d'efficaces réseaux marchands et inventer des stratégies pour mener à bien leurs entreprises. Néanmoins, si l'État florentin acquit sa légitimité en Méditerranée, cette période florissante fut de courte durée. En raison des difficultés à trouver des armateurs pour effectuer les voyages et d'un contexte maritime de plus en plus périlleux, chaque année ne vit pas le départ des galées. De fait, un système parallèle continua toujours d'être utilisé par des marchands peu soucieux d'attendre le départ du convoi officiel. Les lignes de navigation furent abandonnées à la fin des années 1470. Cette expérience avait duré une cinquantaine d'années sans jamais avoir atteint le niveau de l'organisation vénitienne.

Le grand commerce florentin reposait donc sur d'efficaces réseaux marchands qui parcouraient les espaces européen, méditerranéen et atlantique, et auxquels se greffaient une multitude de réseaux locaux sur lesquels les Florentins s'appuyaient pour atteindre des espaces commerciaux qu'ils ne pouvaient fréquenter directement.

lointain, de la Méditerranée à l'océan Indien au XVIII siècle, Paris, 2016; Religion and Trade. CrossCultural Exchanges in World History 1000-1900, éd. F. Trivellato, L. Halevi, C. Antunes, New York, 2014.

2. M. M. Mallett, The Florentine Galleys in the Fifteenth Century, Oxford, 1967.

3. I. Houssaye MichienzI, "La "nation" et les milieux d'affaires florentins aux XIV et XVe siècles", Nation et nations au Moyen Âge. Actes du XLIVe Congrès de la SHMESP, Paris, 2014, p. 299-310. 


\section{Les négociants florentins}

\section{et le commerce transsaharien}

Quelques rares sources, issues du fonds Datini des archives de Prato, illustrent la participation de l'Afrique subsaharienne aux grands courants commerciaux intercontinentaux, exploitant ses propres ressources, parmi lesquelles l'or aurait dû prendre ici une place de choix. Cependant il ne sera pas question d'or, car les sources n'en révèlent pas la présence, mais surtout de cuivre, de plumes d'autruche, de dattes et de maniguette ${ }^{4}$. La documentation ne permet pas de saisir l'intégralité de la chaîne commerciale remontant du bassin du Niger aux côtes européennes, mais, grâce à quelques données, il est possible de saisir un fragment de cette histoire, et de mettre en lumière le rôle de relais des populations juives et converties dans ces échanges, à l'extrême fin du XIV et au début du XV siècle.

L'Afrique saharienne et subsaharienne n'était ni un lieu connu des marchands florentins, ni un objectif commercial. Leurs écrits montrent qu'ils saisirent des opportunités grâce à des relations personnelles de confiance tissées à Ciutat de Majorque ${ }^{5}$ avec des juifs et des juifs convertis. À l'exception de cas isolés, il faudra en effet attendre l'expansion portugaise pour voir les premiers marchands européens pénétrer ces espaces éloignés. Du côté italien, les premières descriptions détaillées sont celles du Génois Antonio Malfante en 1447, qui écrivit une lettre de Tamentit dans le Touat, puis du Florentin Benedetto Dei, qui aurait été le premier Européen à se rendre à Tombouctou vers $1470^{6}$. Les agents de la compagnie Datini ne posèrent, quant à eux, jamais le pied sur le continent africain, mais commerçaient avec l'Afrique à partir de leur bottega de Ciutat de Majorque.

L'île de Majorque, en raison de sa position géographique et de son histoire, en était en effet la voie d'accès privilégiée. La présence à Ciutat d'une importante population juive et nouvellement convertie représentait

4. La maniguette, appelée également poivre de Guinée ou poivre du paradis, est une plante vivace produisant une gousse brune contenant de petites graines utilisées comme condiment et possédant le piquant du poivre.

5. L'actuelle Palma.

6. La lettre que Malfante écrivit du Touat, adressée à Giovanni Marione, découverte par Charles de La Roncière en 1918, est traduite et commentée par ce même auteur dans La découverte de l'Afrique au Moyen Âge, Le Caire, 3 vol., 1925-1927, vol. 1, p. 143-160. À la fin du Xve siècle, le Florentin Benedetto Dei nota, quant à lui, le chemin qu'il avait suivi pour se rendre en Afrique : B. DeI, La cronica dall'anno 1400 all'anno 1500, éd. R. BARducCI, Florence, 1985. Voir également F.-X. Fauvelle, Le rhinocéros d'or, Paris, 2012, chap. 32. 
un atout supplémentaire ${ }^{7}$. Leurs connexions avec les juifs nord-africains, qui furent renforcées lors de la large émigration des juifs majorquins vers le Maghreb après les persécutions de $1391^{8}$, attestaient l'existence de réseaux d'affaires éparpillés entre les deux côtes de la Méditerranée. Les routes maritimes entre l'île de Majorque et les côtes nord-africaines, en particulier avec le Maghreb central et occidental, étaient aux mains de ligues de marchands majorquins qui cherchaient à monopoliser le commerce avec cette région du monde. Ces ligues, dans lesquelles se côtoyaient aussi bien des chrétiens, des juifs et des convertis - seule étant nécessaire la citoyenneté majorquine -, empêchaient de fait les Florentins, dépourvus de flotte, de mener des opérations commerciales en Afrique. En dépit de diverses tentatives, toujours infructueuses, jamais un agent de la compagnie Datini ne débarqua sur les côtes africaines. Par conséquent, le commerce avec le Maghreb fut toujours effectué par le biais d'intermédiaires qui convoyaient et vendaient les marchandises florentines, et ramenaient à Ciutat les articles africains convoités. La plupart d'entre eux étaient des juifs de Majorque ou d'Afrique du Nord, et des convertis qui réceptionnaient les marchandises à Ciutat et les revendaient ensuite aux agents de la compagnie Datini'.

Les opérations suivantes, en plus de démontrer l'existence d'un commerce aux confins du monde africain alors connu, permettent d'observer de manière très précise la collaboration avec des négociants juifs et convertis opérant avec des agents de la compagnie Datini. Le cuivre faisait ainsi l'objet d'un commerce avec l'Afrique subsaharienne ${ }^{10}$. Conduit de Venise, il était stocké à Majorque avant son expédition à Honein, le port de Tlemcen, au Maghreb, qui servait d'entrepôt majeur éminemment situé au point de départ et d'arrivée des caravanes se dirigeant vers

\footnotetext{
7. I. Houssaye Michienzi, "Coexistence et réseaux de relations à Majorque vers 1400 à travers le témoignage des marchands florentins ", Etre citoyen du monde, 2 : Du cosmopolitisme à l'internationalisme : peut-on être citoyen du monde à l'heure des mondialisations? (XV'-XXP siècles), éd. L. CRIPS, N. Gabriel, M.-L. Pelus-Kaplan, Paris, 2015, p. 27-45.

8. Voir EAD., «Entre Majorque et l'Afrique : configuration de l'espace et réseaux juifs d'après des sources commerciales italiennes (fin XIve-début Xve siècle) ", Revue des études juives, 173/1-2 (2014), p. $139-174$.

9. Archivio di Stato di Prato (désormais ASPo), fonds Datini (désormais Datini), 890, 419874, lettre Majorque-Barcelone, 11/07/1403, fol. 1-v : E gl'è venuto Ia fusta di Barberia, e à portato roba asai a gindei [Une fuste est arrivée de Barbarie et a apporté beaucoup de marchandises aux juifs]. 10. Voir M. M. ElbL, «From Venice to the Tuat: Trans-Saharan Copper Trade and Francesco Datini ", Money, Markets and Trade in Late Medieval Europe. Essays in Honour of John H. A. Munro, éd. L. Armstrong, I. Elbl, M. M. Elbl, Leyde, 2007, p. 411-459.
} 
les oasis nord-sahariennes ${ }^{11}$. Il entamait ensuite la traversée du désert. En 1408, Niccolò di Giovanni Manzuoli, alors directeur de la filiale Datini de Majorque, écrit à ses collègues de Barcelone pour leur expliquer le vol d'une cargaison de cuivre expédiée en Afrique et la demande de rançon qu'ils en attendaient par le biais d'une embarcation venant de Honein :

Ecci lettera da Une [Honein] per via di Valenza che tutto il chovero ch'era ito a Tuet, esendo presso a Tuett a 15 leghe Arabi l'anò tutto preso, che più di 6 dobre valea sanza l'altra roba anò rubato, che mai si richorda tal chosa si facesse; ora la lettera chonta ch'erano in su fare rischatto de' Quinti e del Tosugho ve n'era ito da 200 chintali; questi giudei restanno chon gran dispiacere ma stano a fidanza del rischatto. Per la prima che verà di là saprete tutto. Dice la lettera che Arabi andavano a una terra ivi presso per venderne 12 somate che sono intorno di 50 chintali, e que' della terra l'anò ritenuto che s'indovinarono l'avesono rubato. E anòlo venduto e ghuardono i danari per darli a que' di chi sarano, che choriere n'ànno mandato a Une; or' vedete in quanto dispiacere si vive in questo mondo! Questi erano una chonpagnia di mori che domandando a merchadieri della chanfila [caravane] 100 dobre, non gli voglendo dare loro si misono a fare questa rubagione. Dio per sua piate [sic pour pietà] ce ne mandi migl[i] ore nuove ${ }^{12}$ !

Niccolò Manzuoli décrit ainsi dans ses lettres l'organisation de ces expéditions commerciales, exclusivement entre les mains des juifs, comme l'étaient également les opérations de rachat des marchandises volées ${ }^{13}$. Leur

11. Voir M. A. Bouayed, « Le port de Hunayn, trait d'union entre le Maghreb central et l'Espagne au Moyen Âge ", Relaciones de la Península Ibérica con el Magreb (siglos XIII-XVI), éd. M. García Arenal, M. J. Viguera, Madrid, 1988, p. 325-360 ; M. D. López Pérez, J. I. Padilla Lapuente, "Mallorcan Merchants in the Medieval Maghrib: Mercantile Strategies in the Port of Hunayn in the Mid-Fourteenth Century ", Mediterranean Historical Review, 28/2 (2013), p. 141-165.

12. ASPo, Datini 891, 902475, lettre Majorque-Barcelone, 28/02/1408, fol. 2-v, 3-r : « Nous avons reçu une lettre de Honein, via Valence, nous disant que tout le cuivre qui était parti à Touat, étant à 15 lieues près de Touat, a été pris par des Arabes. Il valait plus de 6 doublons sans les autres choses qui furent volées. On ne se souvient pas qu'une telle chose soit déjà survenue. La lettre raconte qu'ils étaient sur le point de rançonner les 200 quintaux qui appartenaient aux Quinti et à Tosugo. Ces juifs étaient désolés mais confiants dans la rançon. Par la prochaine lettre qui viendra vous serez mieux informés. La lettre raconte que les Arabes se rendaient en un lieu proche pour en vendre 12 somate, ce qui correspond à environ 50 quintaux, et ceux de cet endroit les ont retenus, devinant qu'ils l'avaient volé. Ils l'ont vendu et gardent l'argent pour le remettre à ceux à qui cela revient. Ils ont à ce sujet envoyé un courrier à Honein. Voyez dans quelle désolation nous vivons dans ce monde! Ces derniers étaient une compagnie de Maures qui avaient demandé aux marchands de la caravane 100 doublons; ceux-ci ne voulant pas les donner, ils se mirent alors à commettre ce vol. Puisse Dieu, dans sa grande pitié, nous en donner de meilleures nouvelles! »

13. ASPo, Datini 998, 127165, lettre Majorque-Valence, 13/05/1408, fol. 1-r : credesi per questi giudei sia rischattato [nous croyons que par ces juifs il sera rançonné]. 
contact à Honein était un juif dénommé Hayon Sussen, qui était lui-même en relations avec d'autres juifs du Touat ${ }^{14}$ chargés du rachat de la cargaison de cuivre ${ }^{15}$. Ce marchand ne nous est pas inconnu. Il avait investi dans une association commerciale à 2000 livres majorquines de capital, située de part et d'autre de la Méditerranée. Il opérait à Honein et était en partenariat avec son frère Hahim Sussen, basé à Majorque, avec Astruch Xibili, riche négociant juif de Majorque, et avec le gendre de ce dernier, Hahim Xulell, habitant à Tlemcen ${ }^{16}$. Les livres de comptes de la compagnie Datini de Majorque révèlent qu'ils furent d'importants fournisseurs, notamment en produits issus du trafic transsaharien, lui ayant vendu plus de 22000 plumes d'autruche entre 1399 et $1407^{17}$. Hayon Sussen mettait à profit les liens qu'il avait lui-même établis avec des juifs du Touat. Ce sont ainsi ces opérateurs juifs qui établissaient des points de contact essentiels permettant l'écoulement de produits européens jusqu'en Afrique subsaharienne, et l'approvisionnement en articles de ces mêmes régions ${ }^{18}$.

La présence juive dans le Touat est attestée surtout pour la période comprise entre 1300 et $1492^{19}$. Les juifs étaient installés dans les localités situées au débouché des grandes pistes caravanières, se trouvant ainsi au cœur du dispositif commercial de la région, en ces lieux où les caravanes arrivées du Maghreb et celles provenant d'Afrique subsaharienne se

14. Groupe d'oasis du Sahara algérien.

15. ASPo, Datini 892, 902536, lettre Majorque-Barcelone, 16/12/1408, fol. 1-v : è venuto da Une $e$ dice in podere d'Aione Susene, giudeo, à lasciato di nostro e lloro intorno di 315 chintali [...] E'l giudeo che ll'à rischatato è ito chon esso a Tuet [Il est venu de Honein et raconte qu'il a laissé environ 315 quintaux [...] Et le juif qui l'a rançonné est parti avec lui à Touat].

16. M. D. López Pérez, La Corona de Aragón y el Magreb en el siglo XIV (1331-1410), Barcelone, 1995, p. 423.

17. I. Houssaye Michienzi, "Le commerce des plumes d'autruche de l'Afrique subsaharienne aux marchés européens (fin $\mathrm{XIV}^{\mathrm{e}}$-début $\mathrm{XV}^{\mathrm{e}}$ siècle) ", Le commerce du luxe. Production, exposition et circulation des objets précieux du Moyen Âge à nos jours, éd. N. Coquery, A. Bonnet, Paris, 2015, p. 19-26.

18. Au sujet des juifs du Sahara, voir M. Авітвоl, Communautés juives des marges sahariennes du Maghreb, Jérusalem, 1982; H.Z. Hirschberg, "The Problem of the Judaized Berbers ", The Journal of African History, 4/3 (1963), p. 313-339; ID., A History of the Jews in North Africa, Leyde, 1974 ; J. Oliel, Les Juifs au Sahara. Le Touat au Moyen Âge, Paris, 1994; É. Voguet, « Les communautés juives du Maghreb central à la lumière des fatwa-s mālikites de la fin du Moyen Âge ", The Legal Status of dimmi-s in the Islamic West (Second/Eighth-Ninth/fifteenth Centuries), éd. M. Fierro, J.V. Tolan, Turnhout, 2013, p. 295-306.

19. En 1492, la communauté juive du Touat fut détruite et dispersée à la suite des actions menées par le lettré musulman Muhammad al-Magīili. Voir J. Hunwick, Jews of a Saharan Oasis. Elimination of the Tamantit Community, Princeton, 2006. Au sujet du Touat, voir le projet ANR dirigé par Élise Voguet, Le Touat à la croisée des routes sahariennes (XIIT-XVIII siècle): sources, espaces et circulations. 
rencontraient. Le Touat, en plus de posséder d'estimables ressources en eau permettant aux oasis d'accueillir d'importants convois de caravanes, jouissait en effet d'une position privilégiée favorisant le maintien de relations avec des communautés juives établies au Dra, au Tafilalet, au Mzab, avec celles du Maghreb et, à travers elles, avec celles de la péninsule Ibérique et des Baléares. D’après Jacob Oliel, certains commerçants juifs étaient assez riches pour charger leurs propres caravanes ou en commanditer et desservaient la plupart des marchés importants en Afrique subsaharienne, au Sahara et au Maghreb ${ }^{20}$. Ils bénéficiaient de la présence de correspondants juifs à toutes les étapes importantes du commerce, et le pôle TlemcenHonein, qui avait détrôné Sijilmassa, devint la plateforme des échanges entre l'Afrique subsaharienne (via le Touat) et le monde ibérique. Sans divulguer de nouveaux noms, Niccolò Manzuoli fait référence à ces marchands juifs qui circulaient entre Majorque et les côtes maghrébines. Il confia une autre cargaison de cuivre à un juif de Honein qu'il avait certainement rencontré à Ciutat ${ }^{21}$. Ces marchandises, destinées à être vendues dans le Touat, furent également volées, ce qui dévoile un climat d'insécurité constant, déjà dénoncé par Ibn Bațțūța au milieu du XIV ${ }^{e}$ siècle, et toujours virulent en 1447, d'après les affirmations d'Antonio Malfante. Le témoignage des marchands florentins met en lumière une certaine forme de racket. Niccolò Manzuoli affirme, en effet, que la chonpagnia di mori demanda 100 doublons aux marchands de la caravane, qui refusèrent de payer, conduisant ainsi au séquestre des marchandises ${ }^{22}$.

Grâce aux relations de confiance tissées avec les négociants juifs et convertis à Majorque, les agents florentins élargissaient leur horizon. Leur connaissance indirecte de l'aire africaine leur permettait d'envisager des opérations se déroulant bien au-delà de leur base commerciale. Ils remettaient leurs marchandises à des intermédiaires juifs, évoqués d'un œil bienveillant. Hayon Sussen, apprécié de Cristofano di Bartolo (l'ancien directeur de la filiale Datini de Majorque), était qualifié de buono huomo ${ }^{23}$. Son

20. Voir Oliel, Les Juifs..., op. cit. n. 18, p. 56.

21. ASPo, Datini 892, 902536, lettre Majorque-Barcelone, 16/12/1408, fol. 1-v : Apresso fido a uno giudeo di Une chovero per 1256 dobre, él giudeo mandava a Tuet chol'altro e fu rubato tutto [Maintenant je confie à un juif de Honein une cargaison de cuivre d'une valeur de 1256 doublons; le juif l'a envoyée à Touat avec l'autre et il a été entièrement volé].

22. ASPo, Datini 891, 902475, lettre Majorque-Barcelone, 28/02/1408, fol. 2-v, 3-r : Questi erano una chonpagnia di mori che domandando a merchadieri della chanfila 100 dobre, non gli voglendo dare loro si misono a fare questa rubagione [Ces derniers étaient une compagnie de Maures qui avaient demandé aux marchands de la caravane 100 doublons; ceux-ci ne voulant pas les donner, ils se mirent alors à commettre ce vol].

23. ASPo, Datini 892, 902575, lettre Majorque-Barcelone, 31/07/1409, fol. 1-v. 
frère, Hahim Sussen, et Balaix Feraig étaient, quant à eux, définis comme des amici $^{24}$, révélant des liens engageant une certaine forme de solidarité et créant des obligations. Les livres de comptes de la compagnie de Majorque apportent par ailleurs la preuve de l'existence de rapports étroits et quasiment quotidiens entre ces communautés à travers la présence de très nombreuses écritures en caractères hébraïques au sein même de registres de la compagnie destinés à recueillir le témoignage d'une remise d'argent comp$\tan \mathrm{t}^{25}$. De telles écritures, où chacun confirmait de sa main avoir reçu une certaine somme, attestent par exemple la présence d'Hayon Sussen dans la bottega florentine les 4 août, 19 août et 9 septembre 1399, le 20 août 1400 et le 10 décembre 1400. Hahim écrivit, quant à lui, dans les registres Datini les 10 mars et 14 avril 1401 .

Ces relations commerciales devenaient également les vecteurs d'une transmission de connaissances géographiques et culturelles. Les négociants florentins avaient conscience des enjeux du commerce caravanier, qui nécessitait de synchroniser leurs envois avec l'arrivée et le départ des convois. Luca del Sera, de Valence, écrivait ainsi au directeur de la compagnie de Majorque en octobre 1397 qu'il fallait attendre encore deux mois le tenpo della carovana afin que la demande de cuivre augmente ${ }^{26}$. En décembre de la même année, il l'informait que la maniguette arriverait avec les caravanes au mois de janvier ${ }^{27}$. Niccolò Manzuoli utilise pour sa part le terme arabe qäfila, sous la forme chanfila, pour mentionner les caravanes. Les marchands florentins bénéficiaient aussi du savoir géographique véhiculé par les marchands juifs et leurs réseaux d'affaires et dont les cartographes juifs majorquins effectuaient la synthèse, dans l'îlle $e^{28}$ La région du Touat apparaît ainsi dans les lettres de Niccolò Manzuoli, sans explication, ce qui laisse à penser que le destinataire de la lettre (un marchand florentin installé à Barcelone) connaissait l'existence de cette région. Tout comme Antonio Malfante quelques décennies plus tard, Niccolò Manzuoli désigne sous le terme de Touat à la fois une ville et sa région.

24. ASPo, Datini 668, 409993, lettre Majorque-Florence, 07/07/1403, fol. 2-r.

25. I. Houssaye Michienzi, J. Olszowy-Schlanger, «Écrits comptables et commerce interreligieux : les cas des registres d'Ugo Teralh de Forcalquier et de la compagnie Datini (XIVe-Xve siècles) ", Les Cahiers de Framespa [en ligne], 16 (2014), mis en ligne le $1^{\text {er }}$ juillet 2014, consulté le 2 février 2017, http://framespa.revues.org/2917/.

26. ASPo, Datini 1077, 122229, lettre Valence-Majorque, 23/10/1397.

27. Ibid., 122237, lettre Valence-Majorque, 18/12/1397.

28. I. Houssaye Michienzi, E. VAGnon, «Cartographie commerciale et circulations marchandes à Majorque au XVe siècle", Centres pluriculturels et circulation des savoirs (XV'-XXI siècles), éd. S. F. Richer, S. Patin, Paris, 2015, p. 2744. 
De manière très confuse, il identifie aussi des groupes de personnes, en effectuant une éventuelle distinction entre les populations berbères locales désignées sous le terme de mori et les tribus arabes qui s'installaient alors dans la région $\left(\right.$ arabi) ${ }^{29}$.

Si le commerce transsaharien représentait une opportunité commerciale supplémentaire saisie par des marchands florentins utilisant les liens de confiance établis avec les communautés juive et nouvellement convertie, le cas des caravanes de la soie persane était bien différent. Il s'agissait cette fois d'un commerce essentiel pour l'industrie florentine.

\section{Les caravanes de la soie persane}

La nécessité d'importer de plus en plus de soie pour faire fonctionner l'industrie textile florentine à partir du premier tiers $\mathrm{du} \mathrm{Xv}^{\mathrm{e}}$ siècle conduisit à une expansion commerciale des Florentins vers le Levant, d'abord byzantin puis ottoman. Les données comptables de divers ateliers florentins de production de soieries ou de fils d'or révèlent qu'environ un tiers de la soie brute utilisée dans le processus de fabrication à la fin du $X v^{e}$ siècle et au début du Xvi ${ }^{\mathrm{e}}$ provenait de Perse. Vers la fin du Xve siècle, selon les compagnies, la soie persane représentait entre 30 et $40 \%$ des soies brutes utilisées. La compagnie Iacopo Salviati, d'avril 1490 à octobre 1494, durant son premier exercice comptable, acheta plus de 14565 livres florentines de soie brute (près de cinq tonnes ${ }^{30}$ ), dont 4429 livres (une tonne et demie) consistaient en des soies persanes, soit un peu plus de $30 \%$ du total ${ }^{31}$. Toute cette soie était achetée par des agents florentins dépêchés dans l'Empire ottoman, notamment à Brousse (Bursa), au nord-ouest de l'Anatolie, point d'arrivée des caravanes conduisant la précieuse soie depuis le pourtour de la mer Caspienne. L'examen de livres de comptes et de correspondances de négociants florentins présents dans l'Empire ottoman autour de 1500 éclaire la vitalité de ce commerce et montre que les négociants florentins dépendaient des intermédiaires ottomans, notamment des marchands et artisans juifs, pour se procurer la précieuse soie et participer au trafic caravanier.

29. Voir notamment Y. Benhima, Safi et son territoire. Une ville dans son espace au Maroc (11 $16^{e}$ siècle), Paris, 2008, chap. v.

30. La livre de Florence correspondait à un poids de 0,3395 kilogramme.

31. Scuola Normale Superiore di Pisa, archivio Salviati, registres 400 et 409. 
L'exportation de la soie persane commença dès le XiI ${ }^{\mathrm{e}}$ siècle avec l'établissement d'industries de la soie en Italie centrale, mais la présence florentine est attestée dans la région seulement à partir du début du $\mathrm{Xv}^{\mathrm{e}}$ siècle. La soie était importée depuis les provinces du Shirvan, Karabagh, Mazandaran, Khorassan et surtout Gilan de l'Iran timouride puis safavide, sur le littoral de la mer Caspienne ${ }^{32}$. Les sources florentines mentionnent principalement les soies stravai (Asterabad), leggi (Lahijan) et talani (Talish) ${ }^{33}$ qui arrivaient à Brousse après un long parcours caravanier.

Chaque année, en effet, 300 à 400 chameaux convoyaient la ghrande charovana di stravai e di leggi ${ }^{34}$, environ 200 yüks de soie grège, soit plus de 30 tonnes de soie ${ }^{35}$, attendue frénétiquement par les marchands florentins. La voie maritime par la mer Noire de Trébizonde à Constantinople, très active à l'époque byzantine, avait été progressivement abandonnée. De fait, depuis les lieux de production, les caravanes de la soie progressaient vers Erzurum et Erzincan, puis, après le paiement du péage, traversaient l'Anatolie du sud-est au nord-ouest via Sivas et Konya, ou par une route plus au nord passant par Tokat et Amasya ${ }^{36}$. Les conquêtes ottomanes avaient assuré le contrôle des routes de la soie dès le règne du sultan Bajazet $\mathrm{I}^{\mathrm{er}}$.

Ce sont des marchands musulmans de la partie occidentale de l'Iran qui organisaient l'importation de la soie brute, une situation qui perdura jusqu'aux guerres de Sélim I Ir. Une fois à Brousse, les cargaisons étaient déchargées au marché central (bezzäzistān ou "marché aux étoffes") et pesées sur les balances installées dans le caravansérail des cocons (Koza $\left.H_{a n i}\right)^{37}$. Les taxes étaient ensuite prélevées après la pesée par un courtier, le simsār, lui-même contrôlé par un représentant du sultan. Après ces formalités, les marchands caravaniers se voyaient délivrer un certificat sur lequel

32. E. Herzig, "The Iranian Raw Silk Trade and European Manufacture in the Seventeenth and Eighteenth Centuries ", Journal of European Economic History, 19/1 (1990), p. 73-89.

33. Au sujet des provenances des soies alors commercées, voir Francesco Pegolotti, Pratica della mercatura, éd. A. Evans, Cambridge (Mass.), 1936, p. 208 et p. 297-300. Voir également R. P. MatTHEe, The Politics of Trade in Safavid Iran. Silk for Silver 1600-1730, Cambridge, 1999, p. 16 et L. Molì, The Silk Industry of Renaissance Venice, Baltimore, 2000.

34. Archivio di Stato di Firenze, Manoscritti, 94, doc. 18.

35. F. Hitzel, "Production et techniques de tissage de la soie à Bursa aux Xv et $\mathrm{XvI}^{\mathrm{e}}$ siècles ", Rives nord-méditerranéennes [en ligne], 29 (2008), mis en ligne le 21 décembre 2012, consulté le 17 février 2017, http://rives.revues.org/1273. Un yûk correspondait à environ 154 kilogrammes. En 1513, les archives ottomanes signalaient un chargement exceptionnel de 400 yüks, soit plus de 61 tonnes!

36. Ibid., p. 14.

37. O. K. Bagbanci, «Formation of the Historical Commercial Centre in Bursa, the First Capital City of Ottoman Empire », World Applied Sciences Journal, 4/3 (2008), p. 343-348. 
étaient mentionnés le poids de la soie et les droits acquittés, et repartaient avec des produits de fabrication locale ou bien des articles importés par des marchands européens ${ }^{38}$. Parmi ceux-ci dominaient les produits issus des ateliers textiles européens, principalement des industries de la laine.

Les draps de laine florentins (panni) inondaient les marchés ottomans et représentaient la principale monnaie d'échange. Les négociants florentins, qui formaient un groupe de faible importance numérique, avaient néanmoins un intérêt commun : convertir les draps en soie ${ }^{39}$, c'està-dire vendre à Brousse des produits issus de l'industrie textile florentine pour acheter de la soie persane. Giovanni Maringhi, vers 1500, estimait avoir besoin de 600 draps de laine par $\mathrm{an}^{40}$. Aussi encourageait-il en permanence ses partenaires commerciaux de Florence à produire davantage de draps de laine, dont il attendait impatiemment l'arrivée à Pera ${ }^{41}$. Une partie des marchandises réceptionnées à Constantinople était vendue sur place. Une quantité importante prenait toutefois le chemin de Brousse pour y être vendue afin d'alimenter le marché local et de servir de monnaie d'échange, ou dans le but de nouvelles exportations beaucoup plus lointaines, notamment en Perse. Chaque marché était spécifique et avait sa clientèle propre, qui demandait des qualités et des couleurs précises dont les agents florentins se faisaient le relais.

Perché la ttera prop[r]ia ogni giornno vuole 5 ffardelli di seta che li chonsuma, e Chostantinopoli anchora ne chonsuma assai, e Gienovessi ne vogliono somma, e alsi de' nostri anche ne ànno di bisongnio siché chonperatori c'è assai ${ }^{42}$ !

Les caravanes convoyant la soie étaient attendues par une clientèle nombreuse, qui se livrait à une rude concurrence. D'après les dires de Maringhi, les principaux acheteurs de soie étaient les soyeux de Brousse (la terra propria), ceux de Constantinople (Costantinopoli), les Génois (Genovesi) et les Florentins (nostri). Les Vénitiens ne sont pas mentionnés

38. H. Inalcik, An Economic and Social History of the Ottoman Empire 1300-1414, Cambridge, 1994, p. 224 : As Bursa court records from the second half of the fifteenth century demonstrate, Iranian merchants, mostly Muslims from Azerbaijan, exchanged in Bursa their silk loads for Western goods imported by Italians.

39. Business School's Baker Library, Harvard, Selfridge Collection of Medici manuscripts (désormais Selfridge Collection), MS 547, fol. 63-r, mai 1501 : E presto potrebbono diventare setta [Ils pourraient rapidement devenir de la soie].

40. Selfridge Collection, MS 547, fol. 86-v, août 1501.

41. Ibid., fol. 87-v, août 1501 .

42. Ibid., fol. 80-v, juillet 1501 : «Parce que la terre d'ici chaque jour veut 5 fardeaux de soie qu'elle consomme, et Constantinople aussi en consomme beaucoup, et les Génois en veulent une grande quantité, et aussi les nôtres en ont besoin; il y a donc assez d'acheteurs! » 
dans ses lettres, car ils se fournissaient alors en soie à d'autres débouchés caravaniers situés plus au sud, notamment sur les marchés de Damas et $\mathrm{d}^{\prime} \mathrm{Alep}^{43}$. Les lots de soie mis sur le marché partaient très vite. Un agent florentin à Brousse devait être très réactif au marché et avoir les contacts nécessaires pour obtenir d'importantes quantités.

Les lots de soie convoités n'étaient pas directement achetés par les négociants florentins aux caravaniers iraniens ou azéris, mais passaient entre les mains d'intermédiaires qui les vendaient au meilleur prix. D'après les correspondances et les registres de comptes conservés pour la fin du $\mathrm{XV}^{\mathrm{e}}$ et le début du $\mathrm{XVI}^{\mathrm{e}}$ siècle, il s'avère que la soie était achetée principalement auprès des drapiers de Brousse, turcs ou juifs, qui tenaient entre leurs mains le marché de la soie persane. Les sources ne permettent cependant pas de préciser davantage les relations que les Florentins avaient tissées avec ces derniers, se limitant la plupart du temps à des considérations purement commerciales. En septembre 1403, Giovanni Maringhi écrit à un de ses partenaires commerciaux de Florence pour l'informer de l'achat de soie à Brousse auprès d'un intermédiaire juif. Le prix de la soie était de 69 aspres $^{44}$ la livre, comprenant le prix de la soie brute fixé à 61 aspres $1 / 2$ la livre en plus de la commission du marchand juif de 7 aspres $1 / 2$ par livre de soie. Les agents italiens devaient ensuite s'acquitter de droits de douane oscillant entre 2 et $5 \%$.

Questa per dirvi che lla seta vostra l'atendo d'ora in ora di Bursia che doveva partire di Bursia alle XXI di questo sechondo gli avisi, ed è penata tanto a venire perché e'l g[i]udeo à 'uto un pocho di gharbuglio. Pare n'è libero, e chome è detto d'ore in ore può chomparire. E subito chome ci sarà si metterà in chamino e manderà 'viso e chonto e nota de' resto che mi mancherà [...] la quale seta chostò di primo chosto asp. 61 1 $1 / 2$ e l'utile del g[i] udeo sono asp. $71 \frac{1}{2}$ per lib.; in tutto asp. 69 ch'è bonissimo merchato. Chome ci sarà faràssene leghaggio e gitteràssene e'l chonto e tutto vi si manderà ${ }^{45}$.

\footnotetext{
43. Voir Molà, The Silk industry..., op. cit. n. 33; É. Vallet, Marchands vénitiens en Syrie à la fin du XV siècle, Paris, 1999.

44. Il fallait environ 52 aspres pour un florin.

45. Selfridge Collection, MS 547, septembre 1503: "Cette lettre pour vous dire que j'attends d'heure en heure votre soie de Brousse, qui devait partir de Brousse le 21 de ce mois selon les dires, et elle a eu du mal à venir parce que le juif a eu un peu de soucis. Il semble qu'il en est libéré et comme je vous dis d'heure en heure elle peut arriver. Et dès qu'elle sera là elle se mettra en chemin et je vous enverrai la nouvelle et le compte de ce qu'il me manque [...] cette soie coûte de premier coût 61 aspres 1/2, et la commission du juif est de 7 aspres $1 / 2$ par livre; en tout 69 aspres, ce qui est très bon marché. Dès qu'elle sera là j'en ferai l'inventaire et le compte et vous enverrai le tout. »
} 
Les drapiers juifs constituaient également la majorité de la clientèle, qu'ils fussent de Constantinople ou de Brousse. La vente des draps de laine ou panni était au cour du mécanisme d'achat de la soie. Elle permettait d'avoir les moyens d'acheter la soie par troc, paiement différé ou paiement comptant, trois types de paiement que l'on trouve dans la documentation commerciale concernant Brousse. Acheter la soie en paiements différés de plusieurs mois laissait, en effet, aux agents florentins présents sur place le temps de vendre leurs draps de laine. Vente de draps et achats de soie étaient donc étroitement liés et ce sont souvent les mêmes drapiers qui étaient à la fois clients et fournisseurs. La soie était ensuite conditionnée en fardelli, pesant chacun environ 250 livres, soit à peu près 85 kilogrammes. Une fois achetée à Brousse, elle était convoyée à Pera. Il fallait une huitaine de jours pour effectuer le trajet. La précieuse marchandise était toujours acheminée par un marchand florentin. Puis elle prenait essentiellement le chemin de Raguse par les caravanes, puis d'Ancône par voie maritime, avant d'arriver à Florence. La soie pouvait également être conduite à Florence via Lecce, après un trajet caravanier jusqu'aux côtes albanaises, une traversée du canal d'Otrante et une remontée en convoi de mules des Pouilles à Florence. Après un voyage qui pouvait durer six mois et combinait voies de terre et de mer, la soie arrivait enfin à Florence.

Dans les deux cas d'étude présentés, les négociants florentins n'avaient pas accès directement au trafic caravanier et ne pouvaient pas se procurer eux-mêmes la marchandise convoitée, devant ainsi passer par des intermédiaires, ce qui révèle un climat très concurrentiel. Du côté africain, les Majorquins et les Catalans, encouragés par la politique des différents souverains, faisaient barrage aux Florentins en établissant un contrôle quasiment complet des transports entre l'île de Majorque et l'Afrique. Du côté ottoman, la politique sultanienne avait favorisé l'exploitation du commerce intérieur, beaucoup plus considérable que le commerce maritime ${ }^{46}$, par les marchands et artisans ottomans, principalement turcs, juifs et grecs. Les Florentins, arrivés bien plus tardivement que les Génois et les Vénitiens, surent s'imposer sur ces marchés grâce à leur production textile et à la faveur des guerres vénéto-ottomanes, mais le marché intérieur leur était complètement fermé.

46. E. Eldem, "Capitulations and Western trade", The Cambridge History of Turkey, vol. 2, The Ottoman Empire as a World Power, 1453-603, éd. S. FaroqHi, K. Fleet, Cambridge, 2012, p. 281-335. 
Ces deux cas d'étude mettent également en avant des lieux de contact, des places relais. Ciutat de Majorque, où les agents de la compagnie Datini planifiaient depuis leur comptoir les opérations commerciales et confiaient leurs intérêts à des juifs et à des convertis. Le Touat, qui apparaît alors comme une plaque tournante du commerce caravanier transsaharien. Et Brousse, plateforme du commerce de la soie, mais également de celui des épices. Car, parmi la multitude d'artisans et de négociants présents à Brousse, se trouvaient des négociants arabes de Damas et d'Alep, mais également des marchands d'Inde qui y vendaient leurs biens.

Des deux côtés de la Méditerranée, et aux portes du désert du Sahara, les marchands et artisans juifs jouissaient d'une position de force, en tant qu'intermédiaires. Les persécutions de 1391 en péninsule Ibérique, en raison des mouvements migratoires qu'elles provoquèrent en direction de l'Afrique du Nord, avaient renforcé les liens entre les deux rives de la Méditerranée. Leurs réseaux, basés sur d'étroits liens familiaux, étaient répartis de part et d'autre de la mer. Plus tard, l'expulsion de 1492 et la large émigration des juifs vers l'Empire ottoman renforcèrent la position des marchands et artisans juifs, qui devinrent progressivement les intermédiaires indispensables dans le commerce intérieur de l'Empire ottoman, et leur implication en Méditerranée orientale augmenta significativement durant le $\mathrm{XVI}^{\mathrm{e}}$ siècle. Les juifs furent également les seuls, avec les Arméniens, à être partiellement exemptés du blocus commercial imposé par le sultan ottoman Sélim I ${ }^{\text {er }}$ en 1512-1513 sur les caravanes de la soie ${ }^{47}$.

Enfin, ces deux cas d'étude, situés dans des espaces géographique et chronologique distincts, permettent à nouveau de souligner le rôle de groupes de marchands et artisans juifs et convertis dans l'économie internationale. 